\title{
Transition matrix and generalized matrix exponential via the Peano-Baker series
}

\author{
JEFFREY J. DACUNHA* \\ Department of Mathematical Sciences, United States Military Academy, West Point, New York 10996, \\ USA
}

(Received 1 November 2004; in final form 28 June 2005)

\begin{abstract}
We give a closed form for the unique solution to the $n \times n$ regressive time varying linear dynamic system of the form $x^{\Delta}(t)=A(t) x(t), x\left(t_{0}\right)=x_{0}$, via use of a newly developed generalized form of the PeanoBaker series. We develop a power series representation for the generalized time scale matrix exponential when the matrix $A(t) \equiv A$ is a constant matrix. We also introduce a finite series representation of the matrix exponential using the Laplace transform for time scales, as well as a theorem which allows us to write the matrix exponential as a series of $(n-1)$ terms of scalar $C_{r d}^{\infty}(\mathbb{T}, \mathbb{R})$ functions multiplied by powers of the system matrix $A$.
\end{abstract}

Keywords: Peano-Baker series; Time scale; Matrix exponential; Transition matrix; Laplace transformation

1991 Mathematics Subject classification: 39xx

\section{Introduction}

The fact that there exists a unique solution to the regressive linear dynamic system

$$
x^{\Delta}(t)=A(t) x(t), \quad x\left(t_{0}\right)=x_{0}, \quad t_{0} \in \mathbb{T}
$$

on arbitrary time scales $T$ is well-known and this is proven in the excellent introductory text by Bohner and Peterson [4]. However, in their work, there is no closed form given for the solution of equation (1.1). Of course, the common forms are stated for the standard cases of a constant matrix $A(t) \equiv A$ with $\mathbb{T}=\mathbb{R}$ (i.e. $x(t)=e^{A\left(t-t_{0}\right)} x_{0}$ ) and with $\mathbb{T}=h \mathbb{Z}, h>0$, (i.e. $\left.x(t)=(I+h A)^{\left(t-t_{0}\right) / h} x_{0}\right)$.

Adamec [1] has recently made an attempt to derive explicit formulae for the generalized matrix exponential for equation (1.1) when $A(t) \equiv A$ is a constant matrix and the transition matrix of (1.1) for time dependent $A(t)$. The solution is derived by restricting a principal fundamental matrix $Y\left(t, t_{0}\right)$ of an ordinary differential equation $x^{\prime}=H(t) x, t \in \mathbb{R}$. However, the explicit forms of the matrix exponential and transition matrix are only valid if very strong and conservative hypotheses are satisfied-i.e. for the matrix $A(t)$ (resp. $A$ ) and the time scale $\mathbb{T}$, it must be that $\|A(t)\| \cdot\|T\|<1$ (resp. $\|A\| \cdot\|T\|<1$ ), where the first norm is the spectral norm and the second norm is defined to be $\|\mathbb{T}\|:=\sup \{\mu(t): t \in \mathbb{T}\}$.

*Email: jeffrey_dacunha@yahoo.com 
In this paper, we introduce a more general solution to equation (1.1) that has virtually no restrictions on the system matrix $A(t)$ and the time scale of interest. Also, unlike [1] the development remains in the time scales setting throughout the paper. There is no need for carrying over to a corresponding ODE to obtain a principal fundamental matrix, and then bringing that solution back to the time scale domain. In this work, a thorough development with proof is given for a generalized time scales version of the Peano-Baker series, including new generalizations of popular theorems from basic analysis to convergence of sequences and series on intervals in a time scales setting.

We begin where [4] has left off. Section 2 states necessary definitions and theorems are given for use in the derivation of the solution to (1.1). In section 3 , we develop a closed form of the transition matrix for the system in (1.1) on arbitrary time scales via a generalized version of the Peano-Baker series. The formulation of the solution generalizes the classical derivation using Picard iterates [9] for a first order linear differential equation to any time scale. We demonstrate that this closed form of the solution is valid for equation (1.1). From this closed form, section 4 shows that we also garner a generalized power series representation for the matrix exponential on arbitrary time scales. Section 5 first shows, through the use of the generalized Laplace transform [4], that one can obtain a finite series representation of the matrix exponential through a partial fraction expansion of the Laplace transformation of the exponential. Second, we give an existence result which yields an explicit form of the matrix exponential as a finite sum of infinitely rd-continuous delta differentiable functions multiplied by powers of the matrix $A(t)$ from equation (1.1), when $A(t) \equiv A$ is a constant matrix. To conclude, section 6 illustrates the generalized Peano-Baker series in several nontrivial examples to emphasize the legitimacy and utility of this form.

Before beginning, we wish to highlight for the reader the two main themes of the theory of time scales. The first is the unification of the previously disparate cases of continuous differential equations on $\mathbb{R}$ and discrete difference equations on $\mathbb{Z}$. The second, which is of more mathematical and application-oriented interest, is the extension of continuous and discrete analysis to (previously unexplored) arbitrary domains, such as $\mathbb{P}_{a, b}$, the harmonic numbers $\mathbb{H}, q^{\mathbb{Z}}(q>0)$, or something as exotic as the Cantor set $\mathbb{K}$. For a brief summary see the Appendix; for a complete introduction, the reader is referred to $[4,5,6]$.

\section{General definitions}

In this section, we give definitions for certain types of convergence. We also give generalizations of two fundamental theorems from analysis.

Given an infinite series of vector valued functions

$$
\sum_{i=0}^{\infty} x_{i}(t)
$$

with each $x_{i}(t)$ defined on $\left[t_{0}, t_{1}\right]_{\mathbb{T}}$, convergence is defined in terms of the sequence of partial sums

$$
s_{k}(t)=\sum_{i=0}^{k} x_{i}(t)
$$


The series (2.1) converges pointwise to the function $x(t)$ if for each $t \in\left[t_{0}, t_{1}\right]_{\mathbb{T}}$,

$$
\lim _{k \rightarrow \infty}\left\|x(t)-s_{k}(t)\right\|=0 .
$$

The series (2.1) converges uniformly to the function $x(t)$ if, given $\varepsilon>0$, there exists an integer $N_{\varepsilon}>0$ such that for every $t \in\left[t_{0}, t_{1}\right]_{\pi}$,

$$
\left\|x(t)-s_{k}(t)\right\|<\varepsilon, \quad k>N_{\varepsilon} .
$$

We remark that there is an equivalent convergence relationship between the series in (2.1) and the sequence of partial sums $\left\{s_{k}(t)\right\}_{k=0}^{\infty}$. The series (2.1) converges uniformly to $x(t)$ on $\left[t_{0}, t_{1}\right]_{\mathbb{T}}$ if and only if the sequence of partial sums $\left\{s_{k}(t)\right\}_{k=0}^{\infty}$ converges uniformly to $x(t)$ on $\left[t_{0}, t_{1}\right]_{\mathbb{T}}$.

The following theorem is the generalized time scales version of the basic theorem in analysis that states "the uniform limit of continuous functions is continuous."

THEOREM 2.1 (The Uniform Limit of $C_{r d}$ Functions is $C_{r d}$ ). Suppose that equation (2.1) is an infinite series of $r d$-continuous vector valued functions on $\left[t_{0}, t_{1}\right]_{\mathbb{T}}$ that converges uniformly to $x(t)$ on $\left[t_{0}, t_{1}\right]_{\mathbb{T}}$. Then $x(t)$ is rd-continuous for all $t \in\left[t_{0}, t_{1}\right]_{\mathbb{T}}$.

Proof. We need to show that $x(t)$ is continuous for all right dense points in $\left[t_{0}, t_{1}\right]_{\pi}$ and its left-sided limits exists (finite) at all left dense points in $\left[t_{0}, t_{1}\right]_{\mathrm{T}}$.

Choose any $t \in\left[t_{0}, t_{1}\right]_{\mathbb{T}}$ that is right dense and define the sequence of partial sums $s_{k}(t)$ as in (2.2). Given $\varepsilon>0$, we know that there exists an $N_{\varepsilon}>0$ such that, for any $n>N$ and all $t \in\left[t_{0}, t_{1}\right]_{\pi}$, we have $\left\|s_{n}(t)-x(t)\right\|<(\varepsilon / 3)$. Also, since $s_{i}(t) \in \mathrm{C}_{\mathrm{rd}}$ for all $i=0,1, \ldots$ and all $t \in\left[t_{0}, t_{1}\right]_{\mathbb{T}}$, we know that there exists a $\delta>0$ such that for all right dense $t \in\left[t_{0}, t_{1}\right]_{\mathbb{T}}$ and for all $s \in\left[t_{0}, t_{1}\right]_{\mathbb{J}}$ with $0<|t-s|<\delta$, we have $\left\|s_{i}(t)-s_{i}(s)\right\|<\varepsilon / 3$.

We now show that for all right dense $t \in\left[t_{0}, t_{1}\right]_{\pi}$, the limit $x(t)$ of the partial sum is continuous. Given $\varepsilon>0$, for any $s \in\left[t_{0}, t_{1}\right]_{\pi}$ with $0<|t-s|<\delta$, and $n>N$, as above,

$$
\|x(t)-x(s)\| \leq\left\|x(t)-s_{n}(t)\right\|+\left\|s_{n}(t)-s_{n}(s)\right\|+\left\|s_{n}(s)-x(s)\right\|<\frac{\varepsilon}{3}+\frac{\varepsilon}{3}+\frac{\varepsilon}{3}=\varepsilon .
$$

Now choose any $t \in\left[t_{0}, t_{1}\right]_{\text {T }}$ that is left dense and define the sequence of partial sums $s_{k}(t)$ as in equation (2.2). We wish to show that the left hand limit of $x(t)$, denoted $x\left(t^{-}\right)$, exists (finite). Thus, given $\varepsilon>0$, we prove that there exists a $\delta^{*}>0$ such that for all $s, \tau \in$ $\left(t-\delta^{*}, t\right)_{\mathbb{T}}$, we have $\|x(s)-x(\tau)\|<\varepsilon$. Observe, setting $\delta^{*}: \delta$ and $n>N_{\varepsilon}$ from above, given $s, \tau \in\left(t-\delta^{*}, t\right)_{\mathbb{T}} \subset\left[t_{0}, t_{1}\right]_{\mathbb{T}}$ we have

$$
\|x(s)-x(\tau)\| \leq\left\|x(s)-s_{n}(s)\right\|+\left\|s_{n}(s)-s_{n}(\tau)\right\|+\left\|s_{n}(t)-x(\tau)\right\|<\frac{\varepsilon}{3}+\frac{\varepsilon}{3}+\frac{\varepsilon}{3}=\varepsilon
$$

We remark that term-by-term differentiation of a uniformly convergent series of functions does not always give the derivative of the sum. Therefore, we state the next theorem to establish criteria that make this possible.

THEOREM 2.2 Suppose that equation (2.1) is an infinite series of rd-continuouslydifferentiable vector valued functions on $t \in\left[t_{0}, t_{1}\right]_{\mathbb{T}}$ (i.e. $x_{i}(t) \in \mathrm{C}_{\mathrm{rd}}^{1}\left(\left[t_{0}, t_{1}\right]_{\mathbb{\pi}}, \mathbb{R}\right)$ ) for all 
$t \in\left[t_{0}, t_{1}\right]_{\mathbb{J}}$ and all $\left.i \in \mathbb{N}_{0}\right)$ that converges uniformly to $x(t)$ on $\left[t_{0}, t_{1}\right]_{\mathbb{\pi}}$. Then if the series $\sum_{i=0}^{\infty} x_{i}^{\Delta}(t)$ converges uniformly on $\left[t_{0}, t_{1}\right]_{\mathbb{T}}$, it converges to $x^{\Delta}(t)$.

A detailed proof of Theorem 2.2 can be found in [4, Thm. 8.12].

We say that the infinite series (2.1) converges absolutely if the series of real functions $\sum_{i=0}^{\infty}\left\|x_{i}(t)\right\|$ converges on the interval. We will also employ the Weierstrass M-Test in the following section.

Lastly, we ask the reader to refer to the Appendix for the definitions of two types of polynomials used throughout this paper, the generalized polynomial $h_{k}(t, \tau)$ and the generalized $\lambda$-polynomial $p_{k}^{\lambda}(t, \tau)$.

An important fact that will be used in the sequel is that on an arbitrary time scale $\mathbb{T}$, for all $k \in \mathbb{N}_{0}$ and $t \geq t_{0}$, we have $h_{k}\left(t, t_{0}\right) \leq\left(t-t_{0}\right)^{k} / k$ !

\section{The solution to the linear dynamic system}

\subsection{Existence of a solution}

We answer the question of existence of a solution to the regressive homogeneous first order linear dynamic system

$$
x^{\Delta}(t)=A(t) x(t), \quad x\left(t_{0}\right)=x_{0}, \quad t_{0} \in \mathbb{T} .
$$

Given any initial time $t_{0} \in \mathbb{T}$, initial state $x\left(t_{0}\right)=x_{0}$, and an arbitrary time $T>0$, we define a sequence of vectors $\left\{x_{i}(t)\right\}_{i=0}^{\infty}$ on the closed interval $\left[t_{0}, t_{0}+T\right]_{\mathrm{T}}$. This sequence can be thought of as a sequence of approximate solutions to equation (3.1).

We show now that the sequence converges absolutely and uniformly on $\left[t_{0}, t_{0}+T\right]_{\mathrm{T}}$. Define the sequence $\left\{x_{i}(t)\right\}_{i=0}^{\infty}$ on $\left[t_{0}, t_{0}+T\right]_{\mathrm{T}}$ as

$$
\begin{aligned}
x_{0}(t) & =x_{0} \\
x_{1}(t) & =x_{0}+\int_{t_{0}}^{t} A\left(s_{1}\right) x_{0}\left(s_{1}\right) \Delta s_{1} \\
x_{2}(t) & =x_{0}+\int_{t_{0}}^{t} A\left(s_{1}\right) x_{1}\left(s_{1}\right) \Delta s_{1} \\
\vdots & \\
x_{i}(t) & =x_{0}+\int_{t_{0}}^{t} A\left(s_{1}\right) x_{i-1}\left(s_{1}\right) \Delta s_{1} .
\end{aligned}
$$

We can write $x_{i}(t)$ as a sum of terms involving nested integrals of the system matrix $A(t)$,

$$
\begin{aligned}
x_{i}(t)= & x_{0}+\int_{t_{0}}^{t} A\left(s_{1}\right) x_{0} \Delta s_{1}+\int_{t_{0}}^{t} A\left(s_{1}\right) \int_{t_{0}}^{s_{1}} A\left(s_{2}\right) x_{0} \Delta s_{2} \Delta s_{1}+\cdots \\
& +\int_{t_{0}}^{t} A\left(s_{1}\right) \int_{t_{0}}^{t} A\left(s_{2}\right) \cdots \int_{t_{0}}^{s_{i-1}} A\left(s_{i}\right) x_{0} \Delta s_{i} \cdots \Delta s_{1}
\end{aligned}
$$

To analyze the convergence of our sequence, we can write $x_{i}(t)$ as a telescoping sum

$$
x_{i}(t)=x_{0}(t)+\sum_{j=0}^{i-1}\left[x_{j+1}(t)-x_{j}(t)\right], \quad i=0,1, \ldots
$$


This sequence of partial sums of the infinite series of vector functions

$$
x_{0}(t)+\sum_{j=0}^{\infty}\left[x_{j+1}(t)-x_{j}(t)\right]
$$

is exactly the sequence $\left\{x_{i}(t)\right\}_{i=0}^{\infty}$. So we can now analyze the convergence properties of the infinite series in (3.4), since they are equivalent to those of the sequence of vectors in (3.2). We now apply a direct convergent argument to the series.

Since the matrix $A(t) \in \mathrm{C}_{\mathrm{rd}}\left(\mathbb{T}, \mathbb{R}^{n \times n}\right)$ for all $t \in \mathbb{T}$, we see that on the compact interval $\left[t_{0}, t_{0}+T\right]_{\mathrm{T}}$, by $[4$, Thm. 1.65], $\|A(t)\|$ is bounded. Thus we let

$$
\alpha=\max _{t \in\left[t_{0}, t_{0}+T\right]_{\mathrm{T}}}\|A(t)\| \quad \text { and } \quad \beta=\int_{t_{0}}^{t_{0}+T}\left\|A\left(s_{1}\right) x_{0}\right\| \Delta s_{1} .
$$

Now using the terms in the infinite series (3.4),

$$
\left\|x_{1}(t)-x_{0}(t)\right\|=\left\|\int_{t_{0}}^{t} A(s) x_{0} \Delta s_{1}\right\| \leq \int_{t_{0}}^{t}\left\|A(s) x_{0}\right\| \Delta s_{1} \leq \beta, \quad t \in\left[t_{0}, t_{0}+T\right]_{\mathrm{T}} .
$$

Following this we have

$$
\begin{aligned}
\left\|x_{2}(t)-x_{1}(t)\right\| & =\left\|\int_{t_{0}}^{t} A\left(s_{1}\right) x_{1}\left(s_{1}\right)-A\left(s_{1}\right) x_{0}\left(s_{1}\right) \Delta s_{1}\right\| \leq \int_{t_{0}}^{t}\left\|A\left(s_{1}\right)\right\| x_{1}\left(s_{1}\right)-x_{0}\left(s_{1}\right) \| \Delta s_{1} \\
& \leq \int_{t_{0}}^{t} \alpha \beta \Delta s_{1}=\alpha \beta\left(t-t_{0}\right), \quad t \in\left[t_{0}, t_{0}+T\right]_{\mathrm{T}} .
\end{aligned}
$$

In general we have

$$
\begin{aligned}
\left\|x_{i+1}(t)-x_{i}(t)\right\| & =\left\|\int_{t_{0}}^{t} A\left(s_{1}\right) x_{i}\left(s_{1}\right)-A\left(s_{1}\right) x_{i-1}\left(s_{1}\right) \Delta s_{1}\right\| \\
& \leq \int_{t_{0}}^{t}\left\|A\left(s_{1}\right)\right\| x_{i}\left(s_{1}\right)-x_{i-1}\left(s_{1}\right) \| \Delta s_{1} \\
& \leq \beta \frac{\alpha^{i}\left(t-t_{0}\right)^{i}}{i !}, \quad t \in\left[t_{0}, t_{0}+T\right]_{\pi}, \quad i=0,1, \ldots
\end{aligned}
$$

With these bounds, we apply the Weierstrass M-Test. In equation (3.4), the terms are bounded for any $t \in\left[t_{0}, t_{0}+T\right]_{\mathbb{T}}$ since

$$
\left\|x_{0}(t)\right\|=\left\|x_{0}\right\| \quad \text { and } \quad\left\|x_{i+1}(t)-x_{i}(t)\right\| \leq \beta \frac{\alpha^{i} T^{i}}{i !}, \quad i=0,1, \ldots
$$

So the series (3.4) is bounded by a convergent series of bounds

$$
\left\|x_{0}\right\|+\sum_{i=0}^{\infty} \beta \frac{\alpha^{i} T^{i}}{i !}=\left\|x_{0}\right\|+\beta e^{\alpha T} .
$$

So the infinite series (3.4) converges absolutely and uniformly on $\left[t_{0}, t_{0}+T\right]_{\mathbb{T}}$, and since each term in the series is rd-continuous on the interval, by Theorem 2.1 , the limit, which will be denoted $x(t)$, is rd-continuous on the interval. Since the convergence properties of the series in (3.4) are equivalent to the sequence in (3.2), the sequence converges absolutely and uniformly on $\left[t_{0}, t_{0}+T\right]_{\pi}$. 
¿From equation (3.3), letting $i \rightarrow \infty$, the limit of the sequence (3.2) can be written as the infinite series

$$
\begin{aligned}
x(t)= & x_{0}+\int_{t_{0}}^{t} A\left(s_{1}\right) x_{0} \Delta s_{1}+\int_{t_{0}}^{t} A\left(s_{1}\right) \int_{t_{0}}^{s_{1}} A\left(s_{2}\right) x_{0} \Delta s_{2} \Delta s_{1}+\ldots \\
& +\int_{t_{0}}^{t} A\left(s_{1}\right) \int_{t_{0}}^{s_{1}} A\left(s_{2}\right) \ldots \int_{t_{0}}^{s_{i-1}} A\left(s_{i}\right) x_{0} \Delta s_{i} \ldots \Delta s_{1}+\ldots
\end{aligned}
$$

The last step involves showing that this limit $x(t) \in \mathrm{C}_{\mathrm{rd}}^{1}$, and that it satisfies the linear dynamic system (3.1). Evaluating equation (3.6) at $t=t_{0}$, we obtain $x\left(t_{0}\right)=x_{0}$. Delta differentiating equation (3.6) term by term on the right hand side, yields

$$
0+A(t) x_{0}+A(t) \int_{t_{0}}^{t} A\left(s_{2}\right) x_{0} \Delta s_{2}+\cdots+A(t) \int_{t_{0}}^{t} A\left(s_{2}\right) \ldots \int_{t_{0}}^{s_{i-1}} A\left(s_{i}\right) x_{0} \Delta s_{i} \ldots \Delta s_{2}+\ldots
$$

The $i$ th partial sum of equation (3.7) is the $i$ th partial sum of the series $A(t) x(t)$-this can be seen by comparing equation (3.6) to (3.7) - and uniform convergence of (3.7) on $\left[t_{0}, t_{0}+T\right]_{\mathbb{T}}$ follows. By Theorem 2.2, this term by term differentiation yields the delta derivative of $x(t)$ and that derivative is exactly $A(t) x(t)$. We note that since solutions to equation (3.1) are required to be $C_{r d}^{1}$, we note that the terms in (3.7) are $C_{r d}$. We know that by Theorem 2.1, an infinite series of rd-continuous functions that converges uniformly to $x^{\Delta}(t)$ on $\left[t_{0}, t_{0}+T\right]_{\mathrm{T}}$ implies that $x^{\Delta}(t) \in \mathrm{C}_{\mathrm{rd}}$. Thus, we have shown that (3.6) is a solution of equation (3.1). We can use the same process for elements $t \in\left[t_{0}-T, t_{0}\right]_{\mathbb{T}}$, with appropriate uses of absolute value signs in certain inequalities.

Next we factor out the initial vector $x_{0}$ in the series (3.6) to obtain

$$
\begin{aligned}
x(t)= & \left(I+\int_{t_{0}}^{t} A\left(s_{1}\right) \Delta s_{1}+\int_{t_{0}}^{t} A\left(s_{1}\right) \int_{t_{0}}^{s_{1}} A\left(s_{2}\right) \Delta s_{2} \Delta s_{1}\right. \\
& \left.+\cdots+\int_{t_{0}}^{t} A\left(s_{1}\right) \int_{t_{0}}^{s_{1}} A\left(s_{2}\right) \ldots \int_{t_{0}}^{s_{i-1}} A\left(s_{i}\right) \Delta s_{i} \ldots \Delta s_{1}+\ldots\right) x_{0} .
\end{aligned}
$$

We denote the $n \times n$ matrix series in the parentheses of (3.8) by $\Phi_{A}\left(t, t_{0}\right)$-the transition matrix for the system (3.1). We can write our solution to equation (3.1) in terms of the transition matrix as

$$
x(t)=\Phi_{A}\left(t, t_{0}\right) x_{0}
$$

For any $x_{0}$, the $n \times 1$ vector series $\Phi_{A}\left(t, t_{0}\right)$ in equation (3.6) converges absolutely and uniformly at every $t \in\left[t_{0}-T, t_{0}+T\right]_{\pi}$, for any $T>0$. It follows that the $n \times n$ matrix series $\Phi_{A}\left(t, t_{0}\right)$ converges absolutely and uniformly on the same interval. To prove this fact, choose $x_{0}=v_{j}$, the $j$ th column of $I_{n}$, to show the convergence properties of the $j$ th column of $\Phi_{A}\left(t, t_{0}\right)$. 
We may also view this transition matrix as a function of two variables, written as $\Phi_{A}(t, \tau)$, defined by the generalized Peano-Baker series

$$
\begin{aligned}
\Phi_{A}(t, \tau)= & I+\int_{\tau}^{t} A\left(s_{1}\right) \Delta s_{1}+\int_{\tau}^{t} A\left(s_{1}\right) \int_{\tau}^{s_{1}} A\left(s_{2}\right) \Delta s_{2} \Delta s_{1}+\ldots \\
& +\int_{\tau}^{t} A\left(s_{1}\right) \int_{\tau}^{s_{1}} A\left(s_{2}\right) \ldots \int_{\tau}^{s_{i-1}} A\left(s_{i}\right) \Delta s_{i} \ldots \Delta s_{1}+\ldots
\end{aligned}
$$

\subsection{Uniqueness of the solution}

We now show that the there is only one solution to the linear dynamic system (3.1). The strategy is to show that any two solutions of equation (3.1) are necessarily identical.

THEOREM 3.1 (Uniqueness of Solutions) Consider the regressive linear dynamic system (3.1) with arbitrary, but fixed, initial conditions $x\left(t_{0}\right)=x_{0}$. If $x(t), y(t) \in \mathrm{C}_{\mathrm{rd}}^{1}$ are solutions to equation (3.1), then $x(t)=y(t)$ for all $t \in \mathbb{T}$.

Proof. Define $z(t)=x(t)-y(t)$. Then $z(t)$ satisfies

$$
z^{\Delta}(t)=A(t) z(t), \quad z\left(t_{0}\right)=0 .
$$

We now show that (3.10) implies $z(t) \equiv 0$ for all $t \geq t_{0}$.

Integrating both sides of (3.10) from $t_{0}$ to any $t \geq t_{0}$ and taking norms of both sides we have the inequality

$$
\|z(t)\| \leq \int_{t_{0}}^{t}\|A(s)\|\|z(s)\| \Delta s .
$$

Applying the Gronwall's inequality [4], this inequality yields $\|z(t)\|=0$ for all $t \geq t_{0}$.

For $t \leq t_{0}$, a similar development is used which concludes uniqueness of solutions for all $t \in \mathbb{T}$.

THEOREM 3.2 For any initial conditions $x\left(t_{0}\right)=x_{0}$, the regressive linear dynamic system (3.1) has the unique solution

$$
x(t)=\Phi_{A}\left(t, t_{0}\right) x_{0}
$$

where $x(t) \in \mathrm{C}_{\mathrm{rd}}^{1}\left(\mathbb{T}, \mathbb{R}^{n \times n}\right)$.

The transition matrix $\Phi_{A}\left(t, t_{0}\right)$ is given by the Peano-Baker series (3.9) that converges absolutely and uniformly for $t, t_{0} \in[-T, T]$, where $T>0$ is arbitrary.

\section{The constant matrix case}

We now give a special case of when we can simplify the generalized Peano-Baker series into the form of the matrix exponential $e_{A}(t, \tau)$. 
THEOREM 4.1 Suppose that $A(t) \equiv A$ is a constant matrix. Then the transition matrix for equation (3.1) is

$$
\Phi_{A}\left(t, t_{0}\right) \equiv e_{A}\left(t, t_{0}\right)
$$

where, the matrix exponential is defined by the power series

$$
e_{A}\left(t, t_{0}\right)=\sum_{i=0}^{\infty} A^{i} h_{i}\left(t, t_{0}\right)
$$

which converges absolutely and uniformly on $[-T, T]_{\pi}$, for any $T>0$.

Proof. Choose any $T>0$ and set $T^{*}:=2 T$. On any interval $[-T, T]_{\pi}$, the matrix valued function in the series (4.1) are bounded by

$$
\left\|A^{k} h_{k}\left(t, t_{0}\right)\right\|=\left\|A^{k}\right\| \cdot\left|h_{k}\left(t, t_{0}\right)\right| \leq \frac{\left\|A^{k}\right\| \cdot\left|\left(t-t_{0}\right)\right|^{k}}{k !} \leq \frac{\|A\|^{k} T^{*^{k}}}{k !}, \quad k \in \mathbb{N}_{0} .
$$

By the Weierstrass M-Test, since the bounding series of positive real numbers

$$
\sum_{i=0}^{\infty} \frac{\|A\|^{k} T^{*^{k}}}{k !}
$$

converges, the series in (4.1) converges absolutely and uniformly on $[-T, T]$, for any $T>0$

Example 1 In this example, we give insight for the series definition of the matrix exponential in (4.1). From equation (3.1) with $A(t) \equiv A$, a constant matrix, we have the time invariant linear dynamic initial value problem

$$
x^{\Delta}(t)=A x(t), \quad x\left(t_{0}\right)=x_{0}
$$

Using equation (3.2) as an approximating sequence, the general term in the sequence is

$$
\begin{aligned}
x_{i}(t) & =x_{0}+\int_{t_{0}}^{t} A x_{0} \Delta s_{1}+\int_{t_{0}}^{t} A \int_{t_{0}}^{s_{1}} A x_{0} \Delta s_{2} \Delta s_{1}+\cdots+\int_{t_{0}}^{t} A \int_{t_{0}}^{s_{1}} A \ldots \int_{t_{0}}^{s_{i-1}} A x_{0} \Delta s_{i} \ldots \Delta s_{1} \\
& =\left(I+A \int_{t_{0}}^{t} \Delta s_{1}+\cdots+A^{i} \int_{t_{0}}^{t} \int_{t_{0}}^{s_{1}} \ldots \int_{t_{0}}^{s_{i-1}} \Delta s_{i} \ldots \Delta s_{1}\right) x_{0}=\left(\sum_{i=0}^{i} A^{i} h_{i}\left(t, t_{0}\right)\right) x_{0}
\end{aligned}
$$

The limit of this sequence is

$$
\begin{aligned}
x(t) & =\left(I+A \int_{t_{0}}^{t} \Delta s_{1}+\cdots+A^{i} \int_{t_{0}}^{t} \int_{t_{0}}^{s_{1}} \ldots \int_{t_{0}}^{s_{i-1}} \Delta s_{i} \ldots \Delta s_{1}+\ldots\right) x_{0} \\
& =\left(h_{0}\left(t, t_{0}\right)+A h_{1}\left(t, t_{0}\right)+\cdots+A^{i} h_{i}\left(t, t_{0}\right)+\ldots\right) x_{0}=\left(\sum_{i=0}^{\infty} A^{i} h_{i}\left(t, t_{0}\right)\right) x_{0}
\end{aligned}
$$


Delta differentiating equation (4.3) term by term, we obtain

$$
\begin{aligned}
x^{\Delta}(t) & =\left(0+A+A^{2} \int_{t_{0}}^{t} \Delta s_{2}+\cdots+A^{i} \int_{t_{0}}^{t} \int_{t_{0}}^{s_{2}} \ldots \int_{t_{0}}^{s_{i-1}} \Delta s_{i} \ldots \Delta s_{2}+\ldots\right) x_{0} \\
& =A\left(I+A \int_{t_{0}}^{t} \Delta s_{1}+\cdots+A^{i-1} \int_{t_{0}}^{t} \int_{t_{0}}^{s_{1}} \ldots \int_{t_{0}}^{s_{i-2}} x_{0} \Delta s_{i-1} \ldots \Delta s_{1}+\ldots\right) x_{0} \\
& =A\left(I+A h_{1}\left(t, t_{0}\right) \ldots+A^{i-1} h_{i-1}\left(t, t_{0}\right)+\ldots\right) x_{0}=A\left(\sum_{i=0}^{\infty} A^{i} h_{i}\left(t, t_{0}\right)\right) x_{0}=A x(t) .
\end{aligned}
$$

We remark that when $\mathbb{T}=\mathbb{R},(4.3)$ becomes

$$
x(t)=\left(\sum_{i=0}^{\infty} A^{i} h_{i}\left(t, t_{0}\right)\right) x_{0}=\left(\sum_{i=0}^{\infty} \frac{A^{i}\left(t-t_{0}\right)^{i}}{i !}\right) x_{0}=e^{A\left(t-t_{0}\right)} x_{0} .
$$

Thus, from Theorem 4.1 and Example 1, we obtain an infinite series representation for the time scale matrix exponential in (4.1).

\section{Explicit representation of the matrix exponential}

We now develop two explicit representations of the time scale matrix exponential. The first form employs the generalized version of the Laplace transformation [4], while the second shows that the exponential can be expressed as a finite sum of the powers of the matrix constant $A$ in equation (5.1). For the remainder of this section, we consider the regressive matrix initial value problem

$$
X^{\Delta}(t)=A X(t), \quad X\left(t_{0}\right)=I
$$

Taking the Laplace transformation of both sides of equation (5.1) with $t_{0}=0$, we obtain

$$
\mathcal{L}\left\{X^{\Delta}(t)\right\}={ }_{z} \mathcal{L}\{X(t)\}-X(0)=\mathcal{L}\{A X(t)\},
$$

which yields

$$
(z I-A) \mathcal{L}\{X(t)\}=I
$$

and thus

$$
\mathcal{L}\{X(t)\}=(z I-A)^{-1} .
$$

Since we know the unique solution to equation (5.1) is $X(t)=e_{A}(t, 0)$, by the generalized Laplace transform, we see that the Laplace transformation of $e_{A}(t, 0)$ is $(z I-A)^{-1}$. Using the formula

$$
(z I-A)^{-1}=\frac{\operatorname{adj}(z I-A)}{\operatorname{det}(z I-A)},
$$

we see that $(z I-A)^{-1}$ is an $n \times n$ matrix of strictly proper rational functions of $z$. This is true because the det $(z I-A)$ is a degree $n$ polynomial in $z$ while the adj $(z I-A)$ is, at most, an $n-1$ degree polynomial in $z$. 
We can represent the determinant as follows. Letting $\lambda_{1}, \ldots, \lambda_{m}$ be the distinct eigenvalues of $A$ and $s_{1}, \ldots, s_{m}$ be the respective multiplicities (with $m \leq n$ ), we have

$$
\operatorname{det}(z I-A)=\left(z-\lambda_{1}\right)^{s_{1}} \ldots\left(z-\lambda_{m}\right)^{s_{m}}
$$

Thus, the partial fraction expansion of each entry in $(z I-A)^{-1}$ yields

$$
(z I-A)^{-1}=\sum_{k=1}^{m} \sum_{j=1}^{s_{k}} R_{k j} \frac{1}{\left(z-\lambda_{k}\right)^{j}}
$$

where each $R_{k j}$ is an $n \times n$ matrix of partial fraction expansion coefficients (i.e. each entry in $R_{k j}$ is the coefficient of $1 /\left(z-\lambda_{k}\right)^{j}$ in the expansion of the corresponding entry in the matrix $\left.(z I-A)^{-1}\right)$. We note that $R_{k j}$ will be complex valued if the corresponding eigenvalue $\lambda_{k}$ is complex valued. Using a formula for partial fraction expansion coefficients, $R_{k j}$ can be written as

$$
R_{k j}=\left.\frac{1}{\left(s_{k}-j\right) !} \frac{\mathrm{d}^{s_{k}-j}}{\mathrm{~d} z^{s_{k}-j}}\left[\left(z-\lambda_{k}\right)^{s_{k}}(z I-A)^{-1}\right]\right|_{z=\lambda_{k}} .
$$

Taking the inverse Laplace transform, utilizing the Laplace transform in the Appendix, we obtain an explicit representation for the time scale matrix exponential

$$
e_{A}(t, 0)=\sum_{k=1}^{m} \sum_{j=1}^{s_{k}} R_{k j} p_{j-1}^{\lambda_{k}}(t, 0) e_{\lambda_{k}}(t, 0)
$$

The following theorem, motivated by $[7,10]$, shows that we can express the matrix exponential as a finite sum of powers of the matrix $A$ with infinitely rd-continuous delta differentiable functions (i.e. $C_{r d}^{\infty}(\mathbb{T}, \mathbb{R})$ ) as coefficients.

THEOREM 5.1 Consider the matrix linear dynamic IVP (5.1). Then there exist scalar $\mathrm{C}_{\mathrm{rd}}^{\infty}(\mathbb{T}, \mathbb{R})$ functions $\gamma_{0}\left(t, t_{0}\right), \ldots, \gamma_{n-1}\left(t, t_{0}\right)$ such that the unique solution has the representation

$$
e_{A}\left(t, t_{0}\right)=\sum_{k=0}^{n-1} \gamma_{k}\left(t, t_{0}\right) A^{k}
$$

Proof. The strategy we will use is to show that there exist scalar $C_{r d}^{\infty}(\mathbb{T}, \mathbb{R})$ functions $\gamma_{0}\left(t, t_{0}\right), \ldots, \gamma_{n-1}\left(t, t_{0}\right)$ such that

$$
\sum_{k=0}^{n-1} \gamma_{k}^{A}\left(t, t_{0}\right) A^{k}=\sum_{k=0}^{n-1} \gamma_{k}\left(t, t_{0}\right) A^{k+1}, \quad \sum_{k=0}^{n-1} \gamma_{k}\left(t_{0}, t_{0}\right) A^{k}=I
$$

By the Cayley-Hamilton theorem, we have that $A$ satisfies its characteristic polynomial. In other words, we may write

$$
A^{n}=-c_{0} I-c_{1} A-\cdots-c_{n-1} A^{n-1}
$$

where $c_{0}, \ldots, c_{n+1}$ are the coefficients in the characteristic polynomial of $A$. Now we may 
express (5.3) in terms of $I, A, \ldots, A^{n-1}$ as

$$
\begin{aligned}
\sum_{k=0}^{n-1} \gamma_{k}^{\Delta}\left(t, t_{0}\right) A^{k} & =\sum_{k=0}^{n-2} \gamma_{k}^{\Delta}\left(t, t_{0}\right) A^{k+1}-\sum_{k=0}^{n-1} c_{k} \gamma_{n-1}\left(t, t_{0}\right) A^{k} \\
& =-c_{0} \gamma_{n-1}\left(t, t_{0}\right) I+\sum_{k=1}^{n-1}\left[\gamma_{k-1}\left(t, t_{0}\right)-c_{k} \gamma_{n-1}\left(t, t_{0}\right)\right] A^{k}
\end{aligned}
$$

keeping the initial conditions the same.

We solve equation (5.4) by solving the coefficient dynamic equation power of $A$ separately. We obtain the time invariant linear dynamic matrix equation

$$
\left[\begin{array}{c}
\gamma_{0}^{\Delta}\left(t, t_{0}\right) \\
\gamma_{1}^{\Delta}\left(t, t_{0}\right) \\
\vdots \\
\gamma_{n-1}^{\Delta}\left(t, t_{0}\right)
\end{array}\right]=\left[\begin{array}{cccc}
0 & \ldots & 0 & -c_{0} \\
1 & \ldots & 0 & -c_{1} \\
\vdots & \ddots & \vdots & \vdots \\
0 & \ldots & 1 & -c_{n-1}
\end{array}\right]\left[\begin{array}{c}
\gamma_{0}\left(t, t_{0}\right) \\
\gamma_{1}\left(t, t_{0}\right) \\
\vdots \\
\gamma_{n-1}\left(t, t_{0}\right)
\end{array}\right], \quad\left[\begin{array}{c}
\gamma_{0}\left(t_{0}, t_{0}\right) \\
\gamma_{1}\left(t_{0}, t_{0}\right) \\
\vdots \\
\gamma_{n-1}\left(t_{0}, t_{0}\right)
\end{array}\right]=\left[\begin{array}{c}
1 \\
0 \\
\vdots \\
0
\end{array}\right]
$$

Existence of a solution to this linear dynamic matrix equation proves existence of infinitetly rd-continuous delta differentiable functions $\gamma_{0}\left(t, t_{0}\right), \ldots, \gamma_{n-1}\left(t, t_{0}\right)$ that satisfy equation (5.4), thus satisfying equation (5.3).

We present the following simple example to illustrate Theorem 5.1.

Example 2. Consider the regressive linear matrix dynamic system

$$
X^{\Delta}(t)=\left[\begin{array}{cc}
0 & 1 \\
-1 & 0
\end{array}\right] X(t), \quad X\left(t_{0}\right)=I
$$

The characteristic polynomial is $p(\alpha)=\alpha^{2}+1$. Thus, as in Theorem 5.1 , we see that $c_{0}=1$ and $c_{1}=0$. The system we solve is

$$
\left[\begin{array}{c}
\gamma_{0}^{A}\left(t, t_{0}\right) \\
\gamma_{1}^{A}\left(t, t_{0}\right)
\end{array}\right]=\left[\begin{array}{cc}
0 & -1 \\
1 & 0
\end{array}\right]\left[\begin{array}{l}
\gamma_{0}\left(t, t_{0}\right) \\
\gamma_{1}\left(t, t_{0}\right)
\end{array}\right], \quad\left[\begin{array}{l}
\gamma_{0}\left(t_{0}, t_{0}\right) \\
\gamma_{1}\left(t_{0}, t_{0}\right)
\end{array}\right]=\left[\begin{array}{l}
1 \\
0
\end{array}\right]
$$

It is easy to verify that

$$
\gamma_{0}\left(t, t_{0}\right)=\cos _{1}\left(t, t_{0}\right) \text { and } \gamma_{1}\left(t, t_{0}\right)=\sin _{1}\left(t, t_{0}\right)
$$

Thus, we can write the solution $X(t)=e_{A}\left(t, t_{0}\right)$ as the finite series

$$
e_{A}\left(t, t_{0}\right)=\sum_{k=0}^{1} \gamma_{k}\left(t, t_{0}\right) A^{k}=\left[\begin{array}{cc}
\cos _{1}\left(t, t_{0}\right) & 0 \\
0 & \cos _{1}\left(t, t_{0}\right)
\end{array}\right]+\left[\begin{array}{cc}
0 & \sin _{1}\left(t, t_{0}\right) \\
-\sin _{1}\left(t, t_{0}\right) & 0
\end{array}\right]
$$

which is exactly the solution we expected. 


\section{The transition matrix on specific time scales}

We have shown in the first part of this paper how the generalized Peano-Baker series is the unique solution to all first order regressive linear dynamic equations of the form (1.1), (3.1) and (5.1) on arbitrary time scales. In this section, we have chosen some examples to illustrate the Peano-Baker series as the unique solution to the linear dynamic system.

The following example is motivated by [8].

Example 3. Consider the regressive (on $\mathbb{T}=\mathbb{Z}$ ) time varying homogeneous discrete linear dynamic system

$$
\Delta X(t)=A(t) X(t), \quad X(0)=I,
$$

where,

$$
A(t)=\left[\begin{array}{cc}
-1 & \frac{2+(-1)^{t}}{2} \\
\frac{2+(-1)^{t}}{2} & -1
\end{array}\right]
$$

We verify that the transition matrix for equation (6.1) is given by

$$
\Phi_{A}\left(t_{n}, 0\right)=I+\int_{0}^{t_{n}} A\left(s_{1}\right) \Delta s_{1}+\cdots+\int_{0}^{t_{n}} A\left(s_{1}\right) \ldots \int_{0}^{s_{i-1}} A\left(s_{i}\right) \Delta s_{i} \ldots \Delta s_{1}+\ldots,
$$

where, $t_{i}=i \in \mathbb{Z}$. By the composition property of the transition matrix, we have

$$
\Phi_{A}\left(t_{n}, 0\right)=\Phi_{A}\left(t_{n}, t_{n-1}\right) \ldots \Phi_{A}\left(t_{1}, 0\right) .
$$

Thus, for any $1 \leq k \leq n$, by the properties of time scales integration and the fact that $\mu\left(t_{k}\right) \equiv 1$ in $\mathbb{Z}$, we have

$$
\begin{aligned}
\Phi_{A}\left(t_{k}, t_{k-1}\right) & =I+\int_{t_{k-1}}^{t_{k}} A\left(s_{1}\right) \Delta s_{1}+\int_{t_{k-1}}^{t_{k}} A\left(s_{1}\right) \int_{t_{k-1}}^{s_{1}} A\left(s_{2}\right) \Delta s_{2} \Delta s_{1}+\ldots \\
& =I+\mu\left(t_{k-1}\right) A\left(t_{k-1}\right)+\mu\left(t_{k-1}\right) A\left(t_{k-1}\right) \int_{t_{k-1}}^{t_{k-1}} A\left(s_{2}\right) \Delta s_{2}+\ldots \\
& =I+\mu\left(t_{k-1}\right) A\left(t_{k-1}\right)=I+A\left(t_{k-1}\right) .
\end{aligned}
$$

It is clear that after the second term in the Peano-Baker series above, all terms become zero. Thus, with the assumption that an empty product of matrices is the identity matrix,

$$
\Phi_{A}\left(t_{n}, 0\right)=\prod_{i=0}^{t_{n}-1}\left(I+A\left(t_{i}\right)\right)
$$

which becomes

$$
\Phi_{A}\left(t_{n}, 0\right)=\frac{1}{2^{t_{n+1}}}\left[\begin{array}{cc}
(\sqrt{3})^{t_{n}}+(-\sqrt{3})^{t_{n}} & (\sqrt{3})^{t_{n+1}}+(-\sqrt{3})^{t_{n+1}} \\
(\sqrt{3})^{t_{n+1}}+(-\sqrt{3})^{t_{n+1}} & (\sqrt{3})^{t_{n}}+(-\sqrt{3})^{t_{n}}
\end{array}\right] .
$$

In the following example, we show the calculation of the transition matrix via the PeanoBaker series in equation (3.6) produces the solution to the linear dynamic system. 
Example 4. For the $2 \times 2$ matrix linear dynamic system

$$
X^{\Delta}(t)=\left[\begin{array}{ll}
0 & t \\
0 & 0
\end{array}\right] X(t), \quad X\left(t_{0}\right)=\left[\begin{array}{ll}
1 & 0 \\
0 & 1
\end{array}\right]
$$

the transition matrix via the Peano-Baker series is

$$
\Phi_{A}\left(t, t_{0}\right)=\left[\begin{array}{ll}
1 & 0 \\
0 & 1
\end{array}\right]+\int_{t_{0}}^{t}\left[\begin{array}{cc}
0 & s_{1} \\
0 & 0
\end{array}\right] \Delta s_{1}+\int_{t_{0}}^{t}\left[\begin{array}{cc}
0 & s_{1} \\
0 & 0
\end{array}\right] \int_{t_{0}}^{s_{1}}\left[\begin{array}{cc}
0 & s_{2} \\
0 & 0
\end{array}\right] \Delta s_{2} \Delta s_{1}+\ldots
$$

It is easy to see that all terms of the Peano-Baker series are the zero matrix after the second term. Hence,

$$
\Phi_{A}\left(t, t_{0}\right)=\left[\begin{array}{cc}
1 & \int_{t_{0}}^{t} s_{1} \Delta s_{1} \\
0 & 1
\end{array}\right]
$$

The complete solution can be used along with the solution to the homogeneous scalar linear dynamic system

$$
x^{\Delta}(t)=a(t) x(t), \quad x\left(t_{0}\right)=x_{0},
$$

for $t \geq t_{0}$, which is given by $e_{a}\left(t, t_{0}\right) x_{0}$. The next example illustrates a divide and conquer approach to finding the transition matrix which is motivated by [10].

Example 5. To find the solution to the linear dynamic system

$$
x^{\Delta}(t)=\left[\begin{array}{cc}
a(t) & 1 \\
0 & b(t)
\end{array}\right] x(t), \quad x\left(t_{0}\right)=x_{0}=\left[\begin{array}{c}
x_{1_{0}} \\
x_{2_{0}}
\end{array}\right]
$$

we write the system out as a pair of scalar dynamic equations

$$
\begin{aligned}
& x_{1}^{\Delta}(t)=a(t) x_{1}(t)+x_{2}(t), \quad x_{1}\left(t_{0}\right)=x_{1_{0}} \\
& x_{2}^{\Delta}(t)=b(t) x_{2}(t), \quad x_{2}\left(t_{0}\right)=x_{2_{0}} .
\end{aligned}
$$

It is straightforward that $x_{2}(t)=e_{b}\left(t, t_{0}\right) x_{20}$. The first scalar equation can be viewed as the forced scalar dynamic equation

$$
x_{1}^{\Delta}(t)=a(t) x_{1}(t)+e_{b}\left(t, t_{0}\right) x_{2_{0}}, \quad x_{1}\left(t_{0}\right)=x_{1_{0}} .
$$

We can verify the solution is

$$
x_{1}(t)=e_{a}\left(t, t_{0}\right) x_{1_{0}}+\int_{t_{0}}^{t} e_{a}(t, \sigma(s)) e_{b}\left(s, t_{0}\right) x_{2_{0}} \Delta s
$$

Putting into matrix form, we obtain,

$$
x(t)=\left[\begin{array}{cc}
e_{a}\left(t, t_{0}\right) & \int_{t_{0}}^{t} e_{a}(t, \sigma(s)) e_{b}\left(s, t_{0}\right) \Delta s \\
0 & e_{b}\left(t, t_{0}\right)
\end{array}\right] x_{0}
$$

and immediately obtain $\Phi_{A}\left(t, t_{0}\right)$.

It can be verified that the solution obtained by implementing the Peano-Baker series, along with our knowledge of the series representation of the generalized exponential function in 
equation (4.1), is in fact the series representation of the transition matrix $\Phi_{A}\left(t, t_{0}\right)$ for equation (6.3).

\section{References}

[1] Adamec, L., 2004, A remark on matrix equation $x^{\Delta}=A(t) x$ on small time scales. Journal of Difference Equations and Applications, 10, 1107-1117.

[2] Agarwal, R., Bohner, M., O'Regan, D. and Peterson, A., 2002, Dynamic equations on time scales: a survey. Journal of Computational and Applied Mathematics, 141, 1-26.

[3] Bohner, M. and Peterson, A., 2003, Advances in Dynamic Equations on Time Scales (Boston: Birkhäuser).

[4] Bohner, M. and Peterson, A., 2001, Dynamic Equations on Time Scales: An Introduction with Applications (Boston: Birkhäuser).

[5] Hilger, S., 1990, Analysis on measure chains-a unified approach to continuous and discrete calculus. Results in Mathematics, 18, 18-56.

[6] Hilger, S., 1988, Ein Masskettenkalkül mit Anwendung auf Zentrumsmannigfaltigkeiten, Ph.D. thesis. (Germany: Universität Würzburg).

[7] Kailath, T., 1980, Linear Systems (Englewood Cliffs: Prentice-Hall).

[8] Kelly, W. and Peterson, A., 2001, Difference Equations: An Introduction with Applications (Burlington: Harcourt/Academic Press).

[9] Kelly, W. and Peterson, A., 2004, The Theory of Differential Equations Classical and Qualitative (Upper Saddle River: Pearson Education).

[10] Rugh, W.J., 1996, Linear System Theory (Englewood Cliffs: Prentice-Hall).

\section{Appendix: A time scales primer}

\section{A.1 What are time scales?}

A thorough introduction to dynamic equations on time scales is beyond the scope of this appendix. In short, the theory springs from the 1988 doctoral dissertation of Stefan Hilger [6] that resulted in his seminal paper [5] in 1990.These works aimed to unify and generalize various mathematical concepts from the theories of discrete and continuous dynamical systems. Afterwards, the body of knowledge concerning time scales advanced fairly quickly, culminating in the excellent introductory text by Bohner and Peterson [4] and their more recent advanced monograph [3]. The material in this Appendix is drawn mainly from [4]. A succinct survey on time scales can be found in [2].

A time scale $\mathbb{T}$ is any nonempty closed subset of the real numbers $\mathbb{R}$. Thus time scales can be any of the usual integer subsets (e.g. $\mathbb{Z}$ or $\mathbb{N}$ ), the entire real line $\mathbb{R}$, or any combination of discrete points unioned with continuous intervals. The majority of research on time scales so far has focused on expanding and generalizing the vast suite of tools available to the differential and difference equation theorist. We now briefly outline the portions of the time scales theory that are needed for this paper to be as self-contained as is practically possible.

The forward jump operator of $\mathbb{T}, \sigma(t): \mathbb{T} \rightarrow \mathbb{T}$, is given by $\sigma(t)=\inf _{s \in \mathbb{T}}\{s>t\}$. The backward jump operator of $\mathbb{T}, \rho(t): \mathbb{T} \rightarrow \mathbb{T}$, is given by $\rho(t)=\sup _{s \in \mathbb{T}}\{s<t\}$. The graininess function $\mu(t): \mathbb{T} \rightarrow[0, \infty)$ is given by $\mu(t)=\sigma(t)-t$. Here we adopt the conventions $\inf \emptyset=\sup \mathbb{T}$ (i.e. $\sigma(t)=t$ if $\mathbb{T}$ has a maximum element $t$ ), and $\sup \emptyset=$ inf $\mathbb{T}$ (i.e. $\rho(t)=t$ if $\mathbb{T}$ has a minimum element $t$ ). For notational purposes, 
the intersection of a real interval $[a, b]$ with a time scale $\mathbb{T}$ is denoted by $[a, b] \cap$ $\mathbb{T}:=[a, b]_{\mathbb{T}}$.

A point $t \in \mathbb{T}$ is right-scattered if $\sigma(t)>t$ and right dense if $\sigma(t)=t$. A point $t \in \mathbb{T}$ is leftscattered if $\rho(t)<t$ and left dense if $\rho(t)=t$. If $t$ is both left-scattered and right-scattered, we say $t$ is isolated. If $t$ is both left-dense and right-dense, we say $t$ is dense. The set $T^{\kappa}$ is defined as follows: if $\mathbb{T}$ has a left-scattered maximum $m$, then $\mathbb{T}^{\kappa}=\mathbb{T}-\{m\}$; otherwise, $\mathbb{T}^{\kappa}=\mathbb{T}$. If $f: \mathbb{T} \rightarrow \mathbb{R}$ is a function, then the composition $f(\sigma(t))$ is often denoted by $f^{\sigma}(t)$.

For $f: \mathbb{T} \rightarrow \mathbb{R}$ and $t \in \mathbb{T}^{\kappa}$ define $f^{\Delta}(t)$ as the number (when it exists), with the property that, for any $\varepsilon>0$, there exists a neighborhood $U$ of $t$ such that

$$
\left|[f(\sigma(t))-f(s)]-f^{\Delta}(t)[\sigma(t)-s]\right| \leq \epsilon|\sigma(t)-s|, \quad \forall s \in U
$$

The function $f^{\Delta}: \mathbb{T}^{\kappa} \rightarrow \mathbb{R}$ is called the delta derivative or the Hilger derivative of $f$ on $\mathbb{T}^{\kappa}$. We say $f$ is delta differentiable on $\mathbb{T}^{\kappa}$ provided $f^{\Delta}(t)$ exists for all $t \in \mathbb{T}^{\kappa}$.

The following theorem establishes several important observations regarding delta derivatives.

TheOREM A.1 Suppose $f: \mathbb{T} \rightarrow \mathbb{R}$ and $t \in \mathbb{T}^{\kappa}$.

(i) If $f$ is delta differentiable at then $f$ is continuous at $t$.

(ii) If $f$ is continuous at $t$ and $t$ is right-scattered, then $f$ is delta differentiable at $t$ and $\left[f^{\Delta}(t)=f(\sigma(t))-f(t)\right] / \mu(t)$.

(iii) If $t$ is right-dense, then $f$ is delta differentiable at $t$ if and only if $\lim _{s \rightarrow t}[f(t)-$ $f(s)] /(t-s)$ exists. In this case, $f^{\Delta}(t)=\lim _{s \rightarrow t} f(t)-f(s) / t-s$.

(iv) If $f$ is delta differentiable at $t$, then $f(\sigma(t))=f(t)+\mu(t) f^{\Delta}(t)$.

Note that $f^{\Delta}$ is precisely $f^{\prime}$ from the usual calculus when $\mathbb{T}=\mathbb{R}$. On the other hand, $f^{\Delta}=\Delta f=f(t+1)-f(t)$ (i.e. the forward difference operator) on the time scale $\mathbb{T}=\mathbb{Z}$. These are but two very special (and rather simple) examples of time scales. Moreover, the realms of differential equations and difference equations can now be viewed as but special, particular cases of more general dynamic equations on time scales, i.e. equations involving the delta derivative(s) of some unknown function.

A function $f: \mathbb{T} \rightarrow \mathbb{R}$ is $r d$-continuous if $f$ is continuous at every right dense point $t \in \mathbb{T}$, and its left hand limit exists at each left dense point $t \in \mathbb{T}$. The set of rdcontinuous functions $f: \mathbb{T} \rightarrow \mathbb{R}$ will be denoted by $\mathrm{C}_{\mathrm{rd}}=\mathrm{C}_{\mathrm{rd}}(\mathbb{T})=\mathrm{C}_{\mathrm{rd}}(\mathbb{T}, \mathbb{R})$ A function $F: \mathbb{T} \rightarrow \mathbb{R}$ is called a (delta) antiderivative of $f: \mathbb{T} \rightarrow \mathbb{R}$ provided $F^{\Delta}(t)=f(t)$ holds for all $t \in \mathbb{T}^{\kappa}$. The Cauchy integral or definite integral is given by $\int_{a}^{b} f(t) \Delta t=F(b)-F(a)$, for all $a, b \in \mathbb{T}$, where $F$ is any (delta) antiderivative of $f$. Suppose that sup $\mathbb{T}=\infty$. Then the improper integral is defined to by $\int_{a}^{\infty} f(t) \Delta t=\left.\lim _{b \rightarrow \infty} F(t)\right|_{a} ^{b}$ for all $a \in \mathbb{T}$. We remark that the delta integral can be defined in terms of a Lebesgue type integral [3] or a Riemann integral [4].

\section{THEOREM A.2 (Existence of Antiderivatives).}

(i) Every rd-continuous function has an antiderivative. If $t_{0} \in \mathbb{T}$, then $F(t)=\int_{t_{0}}^{t} f(\tau) \Delta \tau$, $t \in \mathbb{T}$, is an antiderivative of $f$.

(ii) If $f \in \mathrm{C}_{\mathrm{rd}}$ and $t \in \mathbb{T}^{\kappa}$, then $\int_{t}^{\sigma(t)} f(\tau) \Delta \tau=f(t) \mu(t)$.

(iii) Suppose $a, b \in \mathbb{T}$ and $f \in \mathrm{C}_{\mathrm{rd}}$. 
(a) If $\mathbb{T}=\mathbb{R}$, then $\int_{a}^{b} f(t) \Delta t=\int_{a}^{b} f(t) \mathrm{d} t$ (the usual Riemann integral).

(b) If $[a, b]_{\mathbb{T}}$ consists of only isolated points, then

$$
\int_{a}^{b} f(t) \Delta t=\left\{\begin{array}{cc}
\sum_{t \in[a, b)_{\mathrm{\top}}} f(t) \mu(t), & a<b, \\
0, & a=b \\
-\sum_{t \in[b, a)_{\mathrm{\top}}} f(t) \mu(t), & a>b .
\end{array}\right.
$$

The last result above reveals that in the continuous case, $\mathbb{T}=\mathbb{R}$, definite integrals are the usual Riemann integrals from calculus. When $\mathbb{T}=\mathbb{Z}$, definite integrals correspond to definite sums from the difference calculus; see [8].

\section{A.2 The Hilger complex plane}

For $h>0$, define the Hilger complex numbers, the Hilger real axis, the Hilger alternating axis, and the Hilger imaginary circle by

$$
\begin{aligned}
& \mathbb{C}_{h}:=\left\{z \in \mathbb{C}: z \neq-\frac{1}{h}\right\}, \quad \mathbb{R}_{h}:=\left\{z \in \mathbb{R}: z>-\frac{1}{h}\right\}, \\
& \mathbb{A}_{h}:=\left\{z \in \mathbb{R}: z<-\frac{1}{h}\right\}, \quad \mathbb{\nabla}_{h}:=\left\{z \in \mathbb{C}:\left|z+\frac{1}{h}\right|=\frac{1}{h}\right\},
\end{aligned}
$$

respectively. For $h=0$, let $\mathbb{C}_{0}:=\mathbb{C}, \mathbb{R}_{0}:=\mathbb{R}, \mathbb{A}_{0}:=\emptyset$ and $\mathbb{l}_{0}:=i \mathbb{R}$. See figure 1 .

Let $h>0$ and $z \in \mathbb{C}_{h}$. The Hilger real part of $z$ is defined by $\operatorname{Re}_{h}(z):=(|z h+1|-1) / h$, and the Hilger imaginary part of $z$ is defined by $\operatorname{Im}_{h}(z):=(\operatorname{Arg}(z h+1)) / h$, where $\operatorname{Arg}(z)$ denotes the principal argument of $z$ (i.e., $-\pi<\operatorname{Arg}(z) \leq \pi$ ). See figure 1 .

For $h>0$, define the strip $\mathbb{Z}_{h}:=\{z \in \mathbb{C}:-\pi / h<\operatorname{Im}(z) \leq \pi / h\}$, and for $h=0$, set $\mathbb{Z}_{0}:=\mathbb{C}$. Then we can define the cylinder transformation $\xi_{h}: \mathbb{C}_{h} \rightarrow \mathbb{Z}_{h}$ by

$$
\xi_{h}(z)=\frac{1}{h} \log (1+z h), \quad h>0,
$$

where, $\log$ is the principal logarithm function. When $h=0$, we define $\xi_{0}(z)=z$, for all $z \in \mathbb{C}$. It then follows that the inverse cylinder transformation $\xi_{h}^{-1}: \mathbb{Z}_{h} \rightarrow \mathbb{C}_{h}$ is given by

$$
\xi_{h}^{-1}(z)=\frac{e^{z h}-1}{h}
$$
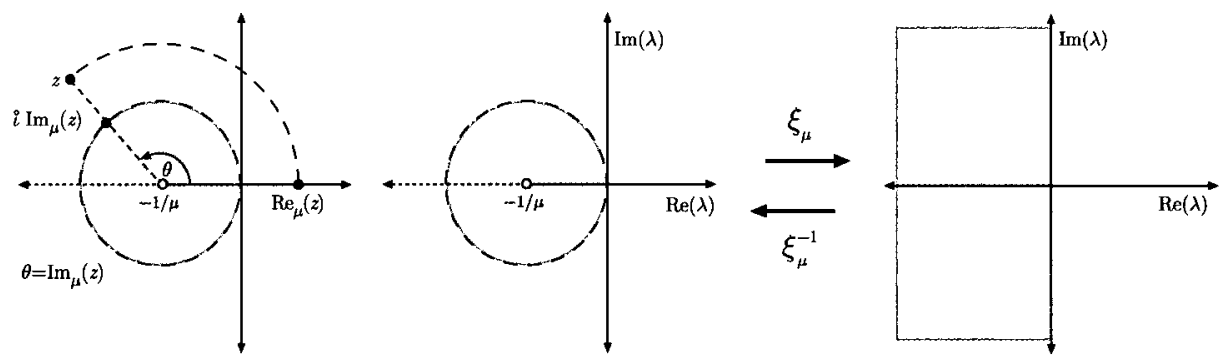

Figure 1. Left: the Hilger complex plane. Right: the cylinder (7.1) and inverse cylinder (7.2) transformations map the familiar stability region in the continuous case to the interior of the Hilger circle in the general time scale case. 
Since the graininess may not be constant for a given time scale, we will interchangeably subscript various quantities (such as $\xi$ and $\xi^{-1}$ ) with $\mu=\mu(t)$ instead of $h$ to reflect this.

\section{A.3 Generalized exponential functions}

The function $p: \mathbb{T} \rightarrow \mathbb{R}$ is regressive if $1+\mu(t) p(t) \neq 0$ for all $t \in \mathbb{T}^{\kappa}$, and this concept motivates the definition of the following sets:

$$
\begin{gathered}
\mathcal{R}=\left\{p: \mathbb{T} \rightarrow \mathbb{R}: p \in \mathrm{C}_{\mathrm{rd}}(\mathbb{T}) \text { and } 1+\mu(t) p(t) \neq 0 \quad \forall t \in \mathbb{T}^{\kappa}\right\} \\
\mathcal{R}^{+}=\left\{p \in \mathcal{R}: 1+\mu(t) p(t)>0 \quad \text { for all } t \in \mathbb{T}^{\kappa}\right\}
\end{gathered}
$$

The function $p: \mathbb{T} \rightarrow \mathbb{R}$ is uniformly regressive on $\mathbb{T}$ if there exists a positive constant $\delta$ such that $0<\delta^{-1} \leq|1+\mu(t) p(t)|, t \in \mathbb{T}^{\kappa}$. A matrix is regressive if and only if all of its eigenvalues are in $\mathcal{R}$. Equivalently, the matrix $A(t)$ is regressive if and only if $I+\mu(t) A$ is invertible for all $t \in \mathbb{T}^{\kappa}$.

If $p \in \mathcal{R}$, then we define the generalized time scale exponential function by

$$
e_{p}(t, s)=\exp \left(\int_{s}^{t} \xi_{\mu(\tau)}(p(\tau)) \Delta \tau\right), \quad \text { for all } s, t \in \mathbb{T} .
$$

The following theorem is a compilation of properties of $e_{p}\left(t, t_{0}\right)$ (some of which are counterintuitive) that we need in the main body of the paper.

THEOREM A.3 The function $e_{p}\left(t, t_{0}\right)$ has the following properties:

(i) If $p \in \mathcal{R}$, then $e_{p}(t, r) e_{p}(r, s)=e_{p}(t, s)$ for all $r, s, t \in \mathbb{T}$.

(ii) $e_{p}(\sigma(t), s)=(1+\mu(t) p(t)) e_{p}(t, s)$.

(iii) If $p \in \mathcal{R}^{+}$then $e_{p}\left(t, t_{0}\right)>0$ for all $t \in \mathbb{T}$.

(iv) If $I+\mu(t) p(t)<0$ for some $t \in \mathbb{T}^{k}$, then $e_{p}\left(t, t_{0}\right) e_{p}\left(\sigma(t), t_{0}\right)<0$.

(v) If $\mathbb{U}=\mathbb{R}$, then $e_{p}(t, s)=e^{\int_{s}^{t} p(\tau) \mathrm{d} \tau}$. Moreover, if $p$ is constant, then $e_{p}(t, s)=e_{p}^{\hat{p} t-s}$.

(vi) If $\mathbb{T}=\mathbb{Z}$, then $e_{p}(t, s)=\prod_{\tau=s}^{t-1}(1+p(\tau))$. Moreover, if $\mathbb{T}=h \mathbb{Z}$, with $h>0$ and $p$ is constant, then $e_{p}(t, s)=(1+h p)^{(t-s) / h}$

If $p \in \mathcal{R}$ and $f: \mathbb{T} \rightarrow \mathbb{R}$ is rd-continuous, then the dynamic equation

$$
y^{\Delta}(t)=p(t) y(t)+f(t)
$$

is called regressive.

THEOREM A.4 (Variation of Constants) Let $t_{0} \in \mathbb{T}$ and $y\left(t_{0}\right)=y_{0} \in \mathbb{R}$. Then the regressive IVP (7.3) has a unique solution $y: \mathbb{T} \rightarrow \mathbb{R}^{n}$ given by

$$
y(t)=y_{0} e_{p}\left(t, t_{0}\right)+\int_{t_{0}}^{t} e_{p}(t, \sigma(\tau)) f(\tau) \Delta \tau .
$$


We say the $n \times 1$-vector-valued system

$$
y^{\Delta}(t)=A(t) y(t)+f(t)
$$

is regressive provided $A \in \mathcal{R}$ and $f: \mathbb{T} \rightarrow \mathbb{R}^{n}$ is a rd-continuous vector-valued function.

Let $t_{0} \in \mathbb{T}$ and assume that $A \in \mathcal{R}$ is an $n \times n$-matrix-valued function. The unique matrixvalued solution to the IVP

$$
Y^{\Delta}(t)=A(t) Y(t), \quad Y\left(t_{0}\right)=I_{n}
$$

where $I_{n}$ is the $n \times n$-identity matrix, is called the transition matrix and it is denoted by $\Phi_{A}\left(t, t_{0}\right)$.

In this paper, we denote the solution to equation (7.5) as $\Phi_{A}\left(t, t_{0}\right)$ when $A(t)$ is time varying and denote the solution as $e_{A}\left(t, t_{0}\right) \equiv \Phi_{A}\left(t, t_{0}\right)$ (the matrix exponential, as in [4]) only when $A(t) \equiv A$ is a constant matrix. Also, if $A(t)$ is a function on $\mathbb{T}$ and the time scale matrix exponential function is a function on some other time scale $\mathbb{S}$, then $A(t)$ is constant with respect to $e_{A(t)}(\tau, s)$, for all $\tau, s \in \mathbb{S}$ and $t \in \mathbb{T}$. The following lemma lists some properties of the transition matrix.

THEOREM A.5 Suppose $A, B \in \mathcal{R}$ are matrix-valued functions on $\mathbb{T}$.

(i) Then the semigroup property $\Phi_{A}(t, r) \Phi_{A}(r, s)=\Phi_{A}(t, s)$ is satisfied for all $r, s, t \in \mathbb{T}$.

(ii) $\Phi_{A}(\sigma(t), s)=(I+\mu(t) A(t)) \Phi_{A}(t, s)$.

(iii) If $\mathbb{T}=\mathbb{R}$ and $A$ is constant, then $\Phi_{A}(t, s)=e_{A}(t, s)=e^{A(t-s)}$.

(iv) If $\mathbb{T}=h \mathbb{Z}$, with $h>0$, and $A$ is constant, then $\Phi_{A}(t, s)=e_{A}(t, s)=(I+h A)^{(t-s) / h}$.

We now present a theorem that guarantees a unique solution to the regressive $n \times 1$-vectorvalued dynamic IVP (7.4).

THEOREM A.6 (Variation of Constants) Let $t_{0} \in \mathbb{T}$ and $y\left(t_{0}\right)=y_{0} \in \mathbb{R}^{n}$. Then the regressive IVP (7.4) has a unique solution $y: \mathbb{T} \rightarrow \mathbb{R}^{n}$ given by

$$
y(t)=\Phi_{A}\left(t, t_{0}\right) y_{0}+\int_{t_{0}}^{t} \Phi_{A}(t, \sigma(\tau)) f(\tau) \Delta \tau .
$$

\section{A.4 Linear systems and regressive matrices}

The following theorem states generalized delta differentiation for matrix-valued functions on arbitrary time scales. Detailed proofs of the following can be found in [4, Thm. 5.2, 5.3].

THEOREM A.7 Suppose that $A$ and $B$ are delta differentiable $n \times n$ matrix-valued functions. Then

(i) $A^{\sigma}(t)=A(t)+\mu(t) A^{\triangle}(t)$;

(ii) $(A+B)^{\Delta}=A^{\Delta}+B^{\Delta}$;

(iii) $(\alpha A)^{\triangle}=\alpha A^{\triangle}$ if $\alpha$ is a constant;

(iv) $(A B)^{\triangle}=A^{\triangle} B+A^{\sigma} B^{\triangle}=A^{\triangle} B^{\sigma}+A B^{\triangle}$;

(v) $\left(A^{-1}\right)^{\triangle}=-\left(A^{\sigma}\right)^{-1} A^{\Delta} A^{-1}=-A^{-1} A^{\Delta}\left(A^{\sigma}\right)^{-1}$ if $A A^{\sigma}$ is invertible. 
We define the matrix-valued function $A(t)$ is regressive (with respect to $\mathbb{T}$ ) provided $I+\mu(t) A(t)$ is invertible for all $t \in \mathbb{T}^{\kappa}$. Equivalently, the matrix $A$ is regressive (with respect to $\mathbb{T}$ ) if and only if each of its eigenvalues are regressive (with respect to $\mathbb{T}$ ).

\section{A.5 A new Laplace transformation}

We start with the definition of the generalized polynomial, which is given by

$$
h_{0}(t, \tau)=1 \quad \text { and } \quad h_{k}(t, \tau)=\int_{\tau}^{t} h_{k-1}(s, \tau) \Delta s
$$

for all $t, \tau \in \mathbb{T}^{\kappa}$ and $k=1,2, \ldots$ The generalized polynomial has delta derivative

$$
h_{0}^{\Delta}(t, \tau)=0 \quad \text { and } \quad h_{k}^{\Delta}(t, \tau)=h_{k-1}(t, \tau)
$$

for all $t, \tau \in \mathbb{T}^{\kappa}$ and $k=1,2, \ldots$

It can be verified that when $\mathbb{T}=\mathbb{R}$, the $k$ th generalized polynomial becomes $h_{k}(t, \tau)=(t-\tau)^{k} / k$ !. When $\mathbb{T}=\mathbb{Z}$, the $k$ th generalized polynomial becomes $h_{k}(t, \tau)=\left(\begin{array}{c}t-\tau \\ k\end{array}\right)$.

We define the generalized $\lambda$-polynomial by

$$
p_{0}^{\lambda}(t, \tau)=1 \quad \text { and } \quad p_{k}^{\lambda}(t, \tau)=\int_{\tau}^{t} \frac{1}{1+\mu(s) \lambda} p_{k-1}^{\lambda}(s, \tau) \Delta s
$$

for all $t, \tau \in \mathbb{T}^{\kappa}$ and $k=1,2, \ldots$

The generalized polynomial has delta derivative

$$
p_{0}^{\lambda}(t, \tau)=0 \quad \text { and } \quad p_{k}^{\lambda}(t, \tau)=\frac{1}{1+\mu(t) \lambda} p_{k-1}^{\lambda}(t, \tau)
$$

for all $t, \tau \in \mathbb{T}^{\kappa}$ and $k=1,2, \ldots$

It can be verified that when $\mathbb{T}=\mathbb{R}$, the $k$ th generalized $\lambda$-polynomial becomes,

$$
p_{k}^{\lambda}(t, \tau)=\frac{(t-\tau)^{k}}{k !}
$$

When $\mathbb{T}=\mathbb{Z}$, the $k$ th generalized $\lambda$-polynomial becomes,

$$
p_{k}^{\lambda}(t, \tau)=\left(\begin{array}{c}
t-\tau \\
k
\end{array}\right) \frac{1}{(1+\lambda)^{k}} .
$$

We say a function $f: \mathbb{T} \rightarrow \mathbb{R}$ is regulated provided its right- and left-sided limits exist at all right- and left-dense points in $\mathbb{T}$, respectively.

The Laplace transformation is given next. Assume that the function $x: \mathbb{T} \rightarrow \mathbb{R}$ is regulated. The Laplace transformation of $x$ is defined by

$$
\mathcal{L}\{x\}(s):=\int_{0}^{\infty} x(t) e_{\ominus s}^{\sigma}(t, 0) \Delta t
$$


for $s \in \mathcal{D}\{x\}$, where $\mathcal{D}\{x\}$ consists of all complex numbers $s$ for which the improper integral exists.

THEOREM A.8 Assume that $p_{k}^{\lambda}(t, 0), k \in \mathbb{N}_{0}$, are defined as above. Then

$$
\mathcal{L}\left\{p_{k}^{\lambda}(t, 0) e_{\lambda}(t, 0)\right\}(s)=\frac{1}{(s-\lambda)^{k+1}}
$$

for those regressive $s \in \mathbb{C}$ satisfying

$$
\lim _{t \rightarrow \infty}\left\{p_{i}^{\lambda}(t, 0) e_{\lambda}(t, 0) e_{\ominus s}(t, 0)\right\}=0, \quad \text { for all } \quad 0 \leq i \leq k
$$


Copyright of Journal of Difference Equations \& Applications is the property of Taylor \& Francis Ltd and its content may not be copied or emailed to multiple sites or posted to a listserv without the copyright holder's express written permission. However, users may print, download, or email articles for individual use. 